(2) Open Access Full Text Article

REVIEW

\title{
Resveratrol as a potential therapeutic drug for respiratory system diseases
}

This article was published in the following Dove Press journal:

Drug Design, Development and Therapy

\section{Xiao-dan Zhu \\ Xiao-ping Lei \\ Wen-bin Dong}

Department of Newborn Medicine, The Affiliated Hospital of Southwest Medical University, Luzhou, Sichuan, People's Republic of China
Correspondence: Wen-bin Dong Department of Newborn Medicine, The Affiliated Hospital of Southwest Medical University, 25 Taiping Road, Luzhou, Sichuan 646000, People's Republic of China

Tel +86 I35 I83897।8

Email dongwenbin2000@।63.com

\begin{abstract}
Respiratory system diseases are common and major ailments that seriously endanger human health. Resveratrol, a polyphenolic phytoalexin, is considered an anti-inflammatory, antioxidant, and anticancer agent. Thanks to its wide range of biological activities, resveratrol has become a hotspot in many fields, including respiratory system diseases. Indeed, research has demonstrated that resveratrol is helpful to relieve pulmonary function in the general population. Meanwhile, growing evidence indicates that resveratrol plays a protective role in respiratory system diseases. This review aimed to summarize the main protective effects of resveratrol in respiratory system diseases, including its anti-inflammatory, antiapoptotic, antioxidant, antifibrotic, antihypertensive, and anticancer activities. We found that resveratrol plays a protective role in the respiratory system through a variety of mechanisms, and so it may become a new drug for the treatment of respiratory system diseases.
\end{abstract}

Keywords: respiratory system diseases, resveratrol, inflammation, apoptosis, oxidation

\section{Introduction}

Resveratrol $\left(3,5,4^{\prime}\right.$-trihydroxy-trans-stilbene) is a polyphenolic compound found in multiple plant species, including grapes and peanuts. Its chemical structure comprises two aromatic rings connected by a methylene bridge; there are two isomeric forms, cis and trans isomers, of which the trans variant displays biological activities. ${ }^{1}$ Resveratrol was first identified by Takaoka in Veratrum grandiflorum roots in 1939. ${ }^{2}$ Later on, it was described as the "French paradox" in 1992, based on the notion that due to red wine consumption, the risk of cardiovascular disease in French individuals is lower than that of other Europeans despite the high intake of saturated fats. ${ }^{2,3}$ In addition to alcohol, red wine contains many polyphenols, including resveratrol. ${ }^{4}$ Multiple studies, including in vitro and animal experiments, have confirmed that resveratrol has protective effects on multiple tissues and organs. ${ }^{5-7}$ This study reviews the potential effects of resveratrol in respiratory system diseases, from the aspects of inflammation, apoptosis, oxidation, fibrosis, pulmonary hypertension, and cancer, describing evidences for related molecular mechanisms (Figure 1).

\section{Anti-inflammatory effects}

Inflammation is an innate protective response of the immune system, resisting external infection and tissue damage, assisting and promoting the recovery of tissue structures, and eliminating invasive pathogens. ${ }^{8}$ However, excessive and uncontrolled inflammation causes severe tissue damage and secondary inflammation, and even DNA damage. ${ }^{8,9} \mathrm{NF}-\kappa \mathrm{B}$, a regulator of the inflammatory process, is essential for maximal transcription of many cytokines that contribute to neutrophil infiltration in the lung. ${ }^{10,11}$ 


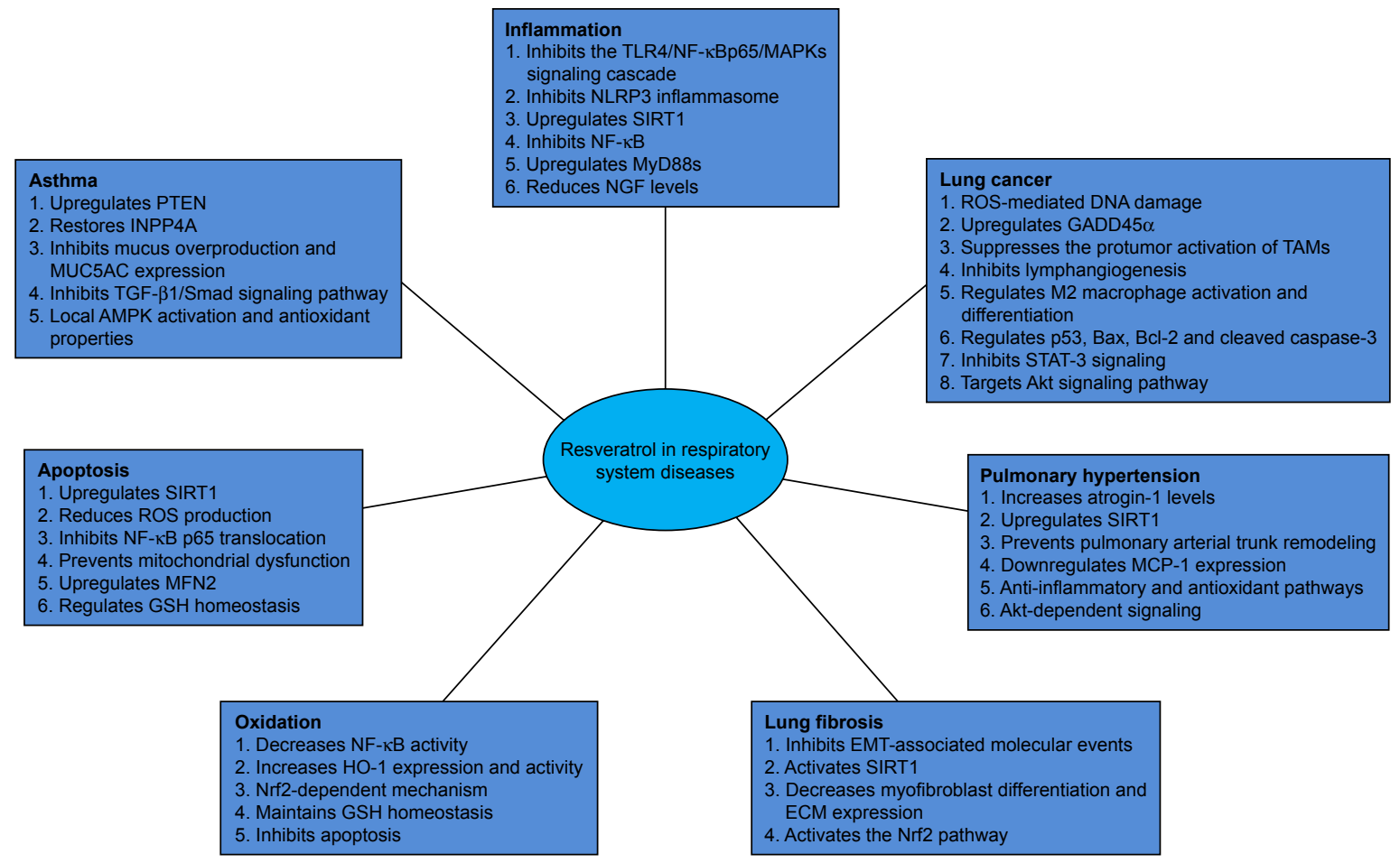

Figure I Protective mechanisms of resveratol in respiratory system diseases.

Abbreviations: ECM, extracellular matrix; EMT, epithelial-mesenchymal transition; GSH, glutathione; ROS, reactive oxygen species; TAM, tumor-associated macrophages.

Therefore, functionally inhibiting NF- $\kappa \mathrm{B}$ is considered a promising anti-inflammatory strategy. SIRT1, an NAD ${ }^{+}$ dependent protein deacetylase, modulates inflammatory responses by deacetylating histones in the promoter regions of downstream genes, including $N F-\kappa B$ and $A P-1 .{ }^{12}$ Resveratrol has protective effects on lipopolysaccharide (LPS)induced inflammation in animal experiments. In the rodent model, resveratrol mitigates LPS-induced acute lung inflam-

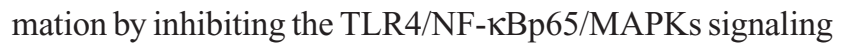
cascade and NLRP3 inflammasome. ${ }^{13,14}$ Resveratrol also alleviates the inhibitory effects of LPS on SIRT1 expression and inhibits LPS-induced activation of MAPKs and NF- $\mathrm{KB}$ both in vivo and in vitro. ${ }^{15}$ Furthermore, resveratrol is helpful in alleviating spinal cord injury-induced inflammatory damage in the rat lung, by significantly decreasing neutrophil infiltration and the production of inflammatory mediators, upregulating SIRT1, and inhibiting NF-אB. ${ }^{16}$ Moreover, resveratrol also reduces the production of inflammatory mediators in respiratory epithelial cells and the lung tissue of mice infected with nontypeable Haemophilus influenzae, by upregulating MyD88s, which is a negative regulator of the NF- $\kappa B$-dependent inflammatory response. ${ }^{17}$ Resveratrol inhibits airway hyperresponsiveness and persistent airway inflammation by reducing NGF levels in respiratory syncytial virus-infected mice. ${ }^{18}$ Resveratrol triggers multiple anti-inflammatory pathways to reduce lung injury caused by staphylococcal enterotoxin B in mice. ${ }^{19}$ In preterm rats, resveratrol protects against hyperoxia-induced lung injury via its antioxidant, anti-inflammatory, and antifibrotic effects, promoting the transdifferentiation of alveolar type II epithelial cells into type I counterparts and suppressing Wnt/ $\beta$-catenin signaling. ${ }^{20}$ Furthermore, resveratrol was shown to attenuate hypoxia-reoxygenation-induced type II pneumocyte dysfunction, partially by promoting surfactant protein expression and suppressing inflammation via NF- $\mathrm{KB}$ pathway involvement. ${ }^{21}$

Asthma is one of the most common chronic inflammatory diseases worldwide, and it is characterized by airway inflammation, remodeling, and airway hyperreponsiveness. Interestingly, resveratrol significantly alleviates asthma by upregulating PTEN, ${ }^{22}$ restoring INPP4A,${ }^{23}$ inhibiting mucus overproduction and MUC5AC expression, ${ }^{24}$ and downregulating TGF- $\beta 1$, inhibiting TGF- $\beta 1 /$ Smad signaling and epithelial-mesenchymal transition (EMT) in an ovalbumininduced murine model of asthma. ${ }^{25}$ In a mouse model of house dust mite-induced asthma, the effects of resveratrol may be associated with Syk protein downregulation. ${ }^{26}$ Another report demonstrated the protective effects of resveratrol in obesityassociated allergic airway inflammation in mice via local AMPK activation and antioxidant properties. ${ }^{27}$ 


\section{Antiapoptotic activities}

Mounting evidence indicates that apoptosis dysregulation is involved in the pathogenetic mechanisms of multiple respiratory system diseases, including asthma, airway inflammation, acute lung injury (ALI), acute respiratory distress syndrome, pulmonary fibrosis, and lung cancer. ${ }^{28-31}$ Reactive oxygen species (ROS) are natural byproducts of cellular metabolism, and induce cell death at excessive levels. ${ }^{32}$ SIRT1 reduces ROS levels and promotes cell activity. ${ }^{33}$ Resveratrol upregulates SIRT1 in human pulmonary alveolar epithelial cells, reduces ROS production, maintains the cell membrane potential, and inhibits apoptosis in alveolar epithelial cells, thus reducing hyperoxia-associated lung injury. ${ }^{34}$ Resveratrol alleviates lung injury and apoptosis in paraquat-induced ALI in mice, via inhibition of NF- $\mathrm{KB}$ p 65 translocation and cytokine production. ${ }^{35}$ Protective effects of resveratrol were also shown in a rat model of chronic obstructive pulmonary disease (COPD) induced by cigarette smoke (CS) exposure combined with intratracheal injection of LPS, via reduction of endoplasmic reticulum stress-induced apoptosis in alveolar epithelial cells. ${ }^{36}$ Moreover, resveratrol exhibits protective effects against CS extract-induced human bronchial epithelial cell apoptosis in vitro by preventing mitochondrial dysfunction and upregulating MFN2. ${ }^{37}$ Vam3, a resveratrol dimer extracted from Vitis amurensis Rupr, also inhibits CSinduced apoptosis in the lung by improving mitochondrial function both in vitro and in vivo. ${ }^{38}$ Resveratrol helps reduce sodium arsenite $\left(\mathrm{NaAsO}_{2}\right)$-induced oxidative and genetic damage as well as apoptosis by regulating glutathione homeostasis in human bronchial epithelial cells. ${ }^{39}$ A previous study showed that resveratrol protects lung function by inhibiting apoptosis in rats with severe acute pancreatitis. ${ }^{40}$ Another report demonstrated that resveratrol attenuates apoptosis in pulmonary microvascular endothelial cells induced by high shear stress with proinflammatory factors, by activating the SIRT1 signaling pathway and inhibiting oxidative-stressdependent phenotypical shift in vitro. ${ }^{41}$

\section{Antioxidant effects}

Oxidative stress is widely recognized to cause lung inflammation, which in turn leads to many lung diseases such as ALI, idiopathic fibrosis, pneumonia, asthma, and COPD. ${ }^{42}$ Resveratrol was shown to exert protective effects against hyperoxic-lung injury via its anti-inflammatory and antioxidant properties in neonatal rats. ${ }^{43}$ A protective effect of resveratrol was also shown in CS-induced lung oxidative injury in mice via decreased NF- $\kappa B$ activity and increased HO-1 expression and activity. ${ }^{44}$ In vitro, resveratrol plays a potential therapeutic role in lung epithelial cells infected with Pseudomonas aeruginosa by reducing ROS formation, ICAM-1 levels, human $\beta$-defensin- 2 expression, and cell apoptosis markers, and upregulating glutathione peroxidase.$^{45}$ In addition, resveratrol attenuates Streptococcus pneumoniae-induced oxidative stress in lung epithelial cells. ${ }^{46}$ Moreover, resveratrol significantly alleviates acute endotoxemia-associated lung injury via reduction of LPSinduced oxidative/nitrative stress in mice. ${ }^{47} \mathrm{It}$ is noteworthy that resveratrol attenuates LPS-induced EMT and pulmonary fibrosis in the murine model by suppressing oxidative stress and TGF- $\beta 1$ signaling. ${ }^{48} \mathrm{HMGB} 1$ is one of the deteriorating factors involved in the development of ventilator-induced lung injury. ${ }^{49}$ Resveratrol exerts protective effects against lung endothelial barrier dysfunction initiated by high tidal volume that involves inhibition of mechanical stretch-induced HMGB1 release and related oxidative damage of mitochondria, likely via an Nrf2-dependent mechanism in a mouse model of lung injury induced by mechanical ventilation. ${ }^{50}$ Another report found that cospray dried resveratrol and budesonide inhalation formulation reduces inflammation and oxidative stress in LPS-induced rat alveolar macrophages. ${ }^{51}$ In in vitro human model of oxidative damage response to arsenic trioxide $\left(\mathrm{As}_{2} \mathrm{O}_{3}\right)$ exposure, resveratrol alleviates oxidative damage by maintaining glutathione homeostasis and inhibiting apoptosis in normal human lung cells. ${ }^{52}$

\section{Antifibrotic effects}

Pulmonary fibrosis is characterized by continuous alveolar epithelial injury and dysregulated repair, which leads to fibroblast accumulation and excessive extracellular matrix deposition. ${ }^{53}$ EMT causes epithelial cells to lose their apical basal polarity and cell-cell adhesion, and convert into mesenchymal cells, which play a potential role in lung fibrosis. ${ }^{54,55}$ Bleomycin (BLM) is an antitumor drug that can cause pulmonary fibrosis during clinical treatment. ${ }^{56}$ Repetitive intratracheal administration of BLM has been used to induce pulmonary fibrosis in animals for many years. ${ }^{57}$ Meanwhile, resveratrol has shown antifibrotic effects in multiple tissues and organs, including the vessels, kidney, and liver in animal models. ${ }^{7,58,59}$ As for the lung, resveratrol has protective effects in BLM-induced lung fibrosis in animals. Resveratrol alleviates the BLM-induced associated pathological changes, weight loss, and mortality in mice, and such effects may be associated with SIRT1 activation and the inhibition of EMTassociated molecular events in the mouse lung. ${ }^{60}$ Similarly, polyphenols exhibit protective effects on the inflammatory process in mice with experimental pulmonary fibrosis. ${ }^{61}$ In an 
established rat model of BLM-induced lung fibrosis, resveratrol also exhibits some therapeutic potential. ${ }^{62}$ A promising therapeutic potential for resveratrol was also demonstrated with decreased myofibroblast differentiation and extracellular matrix expression, and attenuated lung fibrosis in an established mouse model of BLM-associated lung fibrosis. ${ }^{63}$ Furthermore, resveratrol exerts protective effects in paraquatassociated mitochondrial damage, oxidative stress, inflammation, and fibrotic reactions in normal human bronchial epithelial cells by activating the Nrf2 pathway. ${ }^{64}$

\section{Resveratrol reduces pulmonary hypertension}

Pulmonary hypertension is a fatal disease characterized by sustained elevation of pulmonary vascular resistance and a progressive increase in pulmonary artery pressure accompanied by pulmonary vascular remodeling; this results in right ventricular hypertrophy, heart failure, and even death. ${ }^{65,66}$ Monocrotaline (MCT) has been widely applied to induce pulmonary hypertension in animals. ${ }^{67}$ Resveratrol was shown to exert protective effects in pulmonary hypertension both in vivo and in vitro. In vivo, resveratrol decreases MCT-induced pulmonary hypertension indices and normalizes the expression of pulmonary artery atrogin-1 mRNA, which is starkly decreased by MCT in rats. ${ }^{68}$ In vitro, resveratrol significantly inhibits PDGF-induced cell proliferation and hypertrophy, which is also related to increased atrogin-1 levels in human pulmonary artery smooth muscle cells. ${ }^{68}$ In addition, SIRT1 mediates the effects of resveratrol in regulating cell cycle regulatory molecules, contributing to pulmonary hypertension prevention, both in vitro and in vivo. ${ }^{69}$ In a rat model of MCT-induced pulmonary hypertension, resveratrol exerts protective effects by preventing pulmonary arterial trunk remodeling, but not right ventricular hypertrophy. ${ }^{70}$ Moreover, resveratrol inhibits MCT-induced right ventricular hypertrophy, which is mediated by the direct effects of resveratrol on cardiomyocytes as well as the indirect action of reducing pulmonary hypertension in rats. ${ }^{71}$ A protective effect of resveratrol was also demonstrated in pulmonary thromboembolism-induced pulmonary hypertension via downregulation of MCP-1 and inhibition of acute pulmonary thromboembolism-induced p-p38 MAPK activation in rats. ${ }^{72}$ Hypoxic pulmonary hypertension is characterized by progressive pulmonary artery constriction and remodeling. Pulmonary vascular remodeling, which is mainly caused by hypoxia-induced abnormal proliferation of human pulmonary artery smooth muscle cells, leads to sustained increase of pulmonary vascular resistance and hypoxic pulmonary hypertension deterioration. ${ }^{73}$ Resveratrol plays a beneficial role in reducing hypoxic pulmonary hypertension through anti-inflammatory and antioxidant pathways in rats. ${ }^{74}$ Resveratrol was also shown to exert protective effects, by preventing human pulmonary artery smooth muscle cells arginase II induction and proliferation in hypoxic conditions through Akt-dependent signaling. ${ }^{75}$ Moreover, a recent report showed that resveratrol exhibits protective effects by increasing right ventricular systolic pressure and reducing right ventricular hypertrophy in rats exposed to hypoxia alone, via increased SIRT1 activation. ${ }^{76}$ Trimethoxystilbene (TMS, a resveratrol derivative), plays a beneficial role in the treatment of pulmonary hypertension both in vivo and in vitro. In vivo, TMS prevents hypoxia-induced pulmonary vascular remodeling and right ventricular hypertrophy, in association with inhibited NOX/VPO1 pathway as well as inflammatory reaction in rats. ${ }^{77}$ In vitro, TMS inhibits proliferation and apoptosis in pulmonary artery smooth muscle cells stimulated with TNF- $\alpha{ }^{78}$

\section{Anticancer activities}

Lung cancer is one of the most common malignant tumors that seriously endangers human life and health; it is classified as non-small-cell lung cancer (NSCLC, the most common type) and small-cell lung cancer. Despite the recent improvement in lung cancer treatment modalities, such as aggressive radio- and/or chemotherapy, less than $20 \%$ of patients survive for 5 years. ${ }^{79}$ Low-dose resveratrol can inhibit lung cancer cell growth, via premature senescence induced by ROS-mediated DNA damage in NSCLC cells. ${ }^{80}$ In addition, trans-resveratrol induces apoptosis via the mitochondrial-dependent pathway in human lung adenocarcinoma epithelial cells. ${ }^{81}$ Furthermore, resveratrol was shown to exert protective effects in lung cancer cells via modulation of cell apoptosis and proliferation, by binding the synthetic or natural promoter of Egr-1, and upregulating GADD45 $\alpha$ in lung cancer A549 cells. ${ }^{82}$ Resveratrol also effectively inhibits lung cancer growth by restraining the protumor activation of tumor-associated macrophages in human lung cancer. ${ }^{83}$ Furthermore, resveratrol displays antitumor and antimetastatic effects by inhibiting lymphangiogenesis and regulating M2 macrophage activation and differentiation in tumor-associated macrophages. ${ }^{84}$ Moreover, resveratrol inhibits proliferation and induces apoptosis by regulating p53, Bax, Bcl-2, and cleaved caspase-3 in lung adenocarcinoma cells, in a dose-dependent manner. ${ }^{85}$ Resveratrol inhibits lung cancer in a dose-dependent manner both in vitro and in vivo. ${ }^{86}$ In A549 cells, resveratrol exerts protective 
effects against cancer mediated by STAT-3 signaling. ${ }^{87}$ Furthermore, resveratrol inhibits hexokinase II mediated glycolysis by targeting the Akt signaling pathway in NSCLC to suppress tumor growth. ${ }^{88}$ Multiple synthetic resveratrol analogs have been developed in recent years, including 3,4,4'-trihydroxy-trans-stilbene, which induces apoptosis and autophagy in NSCLC cells in vitro. ${ }^{89}$ Studies have shown that resveratrol interacts with RNA to exert its antitumor effects. Interestingly, miR-200c sensitizes $\mathrm{H} 460$ cells to resveratrol, likely via RECK expression. ${ }^{90}$ The novel long noncoding RNA AK001796 is involved in growth inhibition of lung cancer cells induced by resveratrol. ${ }^{91}$ Meanwhile, resveratrol also shows protective effects against lung cancer by inducing considerable changes in the expression profile of microRNAs in A549 cells. ${ }^{92}$ Moreover, resveratrol amplifies the effects of chemotherapy drugs in lung cancer. For example, resveratrol synergizes with gefitinib to increase intracellular concentrations of gefitinib and triggers apoptosis, autophagy, and senescence in gefitinib-resistant NSCLC cells. ${ }^{93}$ In addition, resveratrol combination with cisplatin induces apoptosis by modulating autophagy in A549 cells. ${ }^{94}$ Furthermore, resveratrol and arsenic trioxide synergistically induce apoptosis via ROS mediation of endoplasmic reticulum stress and mitochondrial dysfunction in A549 cells. ${ }^{95,96}$ Moreover, resveratrol also interacts with other chemotherapeutic agents such as etoposide and erlotinib, to exert antitumor effects. ${ }^{79,97}$

\section{Limitations of resveratrol}

In addition to protective effects on the respiratory system, resveratrol also protects against cardiovascular diseases, platelet aggregation, diabetes, and neurodegenerative diseases. The "French paradox" indicates that French individuals have lower incidence of cardiovascular diseases, which raises the question about the amount of wine one should drink to stay healthy. In vitro studies have shown that 111 glasses of wine should be consumed daily to achieve a baseline dose ${ }^{98}$ Despite the benefits of resveratrol in human health, its application in food and pharmaceutical industries is limited for a variety of reasons, including low bioavailability, water solubility, and chemical stability; indeed, this molecule is rapidly and extensively decomposed and excreted. ${ }^{99-101}$ Indeed, the water solubility of resveratrol is too low without emulsifiers or stabilizers to produce the desired concentrations of aqueous solution..$^{99}$ Resveratrol is a highly light-sensitive compound, and exposure to light causes about $80 \%-90 \%$ of trans-resveratrol in solution to convert into cis-resveratrol. ${ }^{102}$ Resveratrol is also easily degraded at high temperature, as well as under $\mathrm{pH}$ change, ambient fluorescent light, or air exposure. ${ }^{101,103}$ Two clinical trials have assessed the absorption and bioavailability of resveratrol, using a single $25 \mathrm{mg}$ oral dose, which is equivalent to a moderate intake of red wine. ${ }^{100,104}$ Despite the use of highly sensitive analytical methods and specific molecules, circulating plasma resveratrol was barely detectable. The low bioavailability of resveratrol is largely due to extensive metabolism in the gut and liver. ${ }^{105}$ Meanwhile, in most studies, resveratrol is used in the free form, which is not suitable for drug delivery. ${ }^{106}$ In recent years, various attempts have been made to improve the oral bioavailability of resveratrol, including complexation with $\beta$-cyclodextrins or hydroxypropyl- $\beta$ cyclodextrins, ${ }^{107}$ solid dispersion, ${ }^{108}$ liposomes, ${ }^{109}$ and lipid or polymeric nanoparticles. ${ }^{106,110}$ Despite important advances, these technologies still face limitations, primarily with the use of large amounts of excipients and insufficient relevant bioavailability data. Therefore, new formulations may be a way to improve resveratrol's bioavailability and target specific organs.

\section{Conclusion}

The protective effects of resveratrol in respiratory system diseases were discussed in terms of anti-inflammatory, antiapoptotic, antioxidant, antifibrotic, antihypertensive, and antitumor properties, demonstrating a great potential for resveratrol in the treatment of respiratory system diseases. So far, only few clinical studies have analyzed the effects of resveratrol, including only a few patients. In addition, existing studies have focused on rodent models, with little efforts invested in animals closely related to humans, such as pigs and nonhuman primates. Therefore, future research should shift the focus to large animals and gradually transfer to clinical trials, while improving the stability and bioavailability of resveratrol. Such studies would provide a strong basis for the use of resveratrol, which has great therapeutic potential.

\section{Acknowledgment}

This work was supported by the National Natural Science Foundation of China (No 81571480 to Wen-bin Dong).

\section{Disclosure}

The authors report no conflict of interest in this work.

\section{References}

1. Oyenihi OR, Oyenihi AB, Adeyanju AA, Oguntibeju OO. Antidiabetic effects of resveratrol: the way forward in its clinical utility. $J$ Diabetes Res. 2016;2016:9737483. 
2. Catalgol B, Batirel S, Taga Y, Ozer NK. Resveratrol: French paradox revisited. Front Pharmacol. 2012;3:141.

3. Renaud S, de Lorgeril M. Wine, alcohol, platelets, and the French paradox for coronary heart disease. Lancet. 1992;339(8808):1523-1526.

4. Cho S, Namkoong K, Shin M, et al. Cardiovascular protective effects and clinical applications of resveratrol. J Med Food. 2017;20(4): 323-334.

5. Vella RK, Pullen C, Coulson FR, Fenning AS. Resveratrol prevents cardiovascular complications in the SHR/STZ rat by reductions in oxidative stress and inflammation. BioMed Res Int. 2015;2015:918123.

6. Sadi G, Pektas MB, Koca HB, Tosun M, Koca T. Resveratrol improves hepatic insulin signaling and reduces the inflammatory response in streptozotocin-induced diabetes. Gene. 2015;570(2):213-220.

7. Zhang H, Sun Q, Xu T, et al. Resveratrol attenuates the progress of liver fibrosis via the Akt/nuclear factor-kappaB pathways. Mol Med Rep. 2016;13(1):224-230.

8. Ma Y, Bao Y, Wang S, et al. Anti-inflammation effects and potential mechanism of saikosaponins by regulating nicotinate and nicotinamide metabolism and arachidonic acid metabolism. Inflammation. 2016;39(4):1453-1461.

9. Arimura K, Aoshiba K, Tsuji T, Tamaoki J. Chronic low-grade systemic inflammation causes DNA damage in the lungs of mice. Lung. 2012; 190(6):613-620.

10. Yang R, Yang L, Shen X, et al. Suppression of NF-kappaB pathway by crocetin contributes to attenuation of lipopolysaccharideinduced acute lung injury in mice. Eur J Pharmacol. 2012;674(2-3): 391-396.

11. Wang J, Liu YT, Xiao L, Zhu L, Wang Q, Yan T. Anti-inflammatory effects of apigenin in lipopolysaccharide-induced inflammatory in acute lung injury by suppressing COX-2 and NF-kB pathway. Inflammation. 2014;37(6):2085-2090.

12. Deng Z, Jin J, Wang Z, Wang Y, Gao Q, Zhao J. The metal nanoparticleinduced inflammatory response is regulated by SIRT1 through NFkappaB deacetylation in aseptic loosening. Int J Nanomed. 2017;12: 3617-3636.

13. Wang G, Hu Z, Fu Q, et al. Resveratrol mitigates lipopolysaccharidemediated acute inflammation in rats by inhibiting the TLR4/NFkappaBp65/MAPKs signaling cascade. Sci Rep. 2017;7:45006.

14. Jiang L, Zhang L, Kang K, et al. Resveratrol ameliorates LPS-induced acute lung injury via NLRP3 inflammasome modulation. Biomed Pharmacother. 2016;84:130-138.

15. Ma L, Zhao Y, Wang R, et al. 3,5,4'-Tri-O-acetylresveratrol attenuates lipopolysaccharide-induced acute respiratory distress syndrome via MAPK/SIRT1 pathway. Mediators Inflamm. 2015;2015:143074.

16. Liu J, Yi L, Xiang Z, Zhong J, Zhang H, Sun T. Resveratrol attenuates spinal cord injury-induced inflammatory damage in rat lungs. Int J Clin Exp Pathol. 2015;8(2):1237-1246.

17. Andrews CS, Matsuyama S, Lee BC, Li JD. Resveratrol suppresses NTHi-induced inflammation via up-regulation of the negative regulator MyD88 short. Sci Rep. 2016;6:34445.

18. Zang N, Li S, Li W, et al. Resveratrol suppresses persistent airway inflammation and hyperresponsivess might partially via nerve growth factor in respiratory syncytial virus-infected mice. Int Immunopharmacol. 2015;28(1):121-128.

19. Rieder SA, Nagarkatti P, Nagarkatti M. Multiple anti-inflammatory pathways triggered by resveratrol lead to amelioration of staphylococcal enterotoxin B-induced lung injury. Br J Pharmacol. 2012;167(6): $1244-1258$.

20. Xu W, Zhao Y, Zhang B, et al. Resveratrol attenuates hyperoxiainduced oxidative stress, inflammation and fibrosis and suppresses Wnt/ $\beta$-catenin signalling in lungs of neonatal rats. Clin Exp Pharmacol Physiol. 2015;42(10):1075-1083.

21. Liu PL, Chong IW, Lee YC, et al. Anti-inflammatory effects of resveratrol on hypoxia/reoxygenation-induced alveolar epithelial cell dysfunction. J Agric Food Chem. 2015;63(43):9480-9487.

22. Chen G, Tang J, Ni Z, et al. Antiasthmatic effects of resveratrol in ovalbumin-induced asthma model mice involved in the upregulation of PTEN. Biol Pharm Bull. 2015;38(4):507-513.
23. Aich J, Mabalirajan U, Ahmad T, et al. Resveratrol attenuates experimental allergic asthma in mice by restoring inositol polyphosphate 4 phosphatase (INPP4A). Int Immunopharmacol. 2012;14(4):438-443.

24. Ni ZH, Tang JH, Chen G, et al. Resveratrol inhibits mucus overproduction and MUC5AC expression in a murine model of asthma. Mol Med Rep. 2016;13(1):287-294.

25. Lee HY, Kim IK, Yoon HK, Kwon SS, Rhee CK, Lee SY. Inhibitory effects of resveratrol on airway remodeling by transforming growth factor-beta/Smad signaling pathway in chronic asthma model. Allergy Asthma Immunol Res. 2017;9(1):25-34.

26. Chen J, Zhou H, Wang J, et al. Therapeutic effects of resveratrol in a mouse model of HDM-induced allergic asthma. Int Immunopharmacol. 2015;25(1):43-48.

27. Andre DM, Calixto MC, Sollon C, et al. Therapy with resveratrol attenuates obesity-associated allergic airway inflammation in mice. Int Immunopharmacol. 2016;38:298-305.

28. Johnson A, DiPietro LA. Apoptosis and angiogenesis: an evolving mechanism for fibrosis. FASEB J. 2013;27(10):3893-3901.

29. Galani V, Tatsaki E, Bai M, et al. The role of apoptosis in the pathophysiology of Acute Respiratory Distress Syndrome (ARDS): an up-to-date cell-specific review. Pathol Res Pract. 2010;206(3):145-150.

30. Walsh GM. Eosinophil apoptosis and clearance in asthma. J Cell Death. 2013;6:17-25.

31. Liu G, Pei F, Yang F, et al. Role of autophagy and apoptosis in nonsmall-cell lung cancer. Int J Mol Sci. 2017;18(2):367.

32. Hori YS, Kuno A, Hosoda R, Horio Y. Regulation of FOXOs and p53 by SIRT1 modulators under oxidative stress. PLoS One. 2013;8(9): e73875.

33. Horio Y, Hayashi T, Kuno A, Kunimoto R. Cellular and molecular effects of sirtuins in health and disease. Clin Sci. 2011;121(5):191-203.

34. Zhang C, Li Q, Kang L, et al. [Resveratrol inhibits hyperxia-induced cell apoptosis through up-regulating SIRT1 expression in HPAECs]. Xi Bao Yu Fen Zi Mian Yi Xue Za Zhi. 2015;31(5):590-595.

35. Zhao G, Li S, Hong G, et al. The effect of resveratrol on paraquatinduced acute lung injury in mice and its mechanism. Zhonghua Wei Zhong Bing Ji Jiu Yi Xue. 2016;28(1):33-37.

36. Li Y, Luo B, Zhang L, Ma M, Guo X. Resveratrol attenuates endoplasmic reticulum stress and alveolar epithelial apoptosis in a rat model of chronic obstructive pulmonary disease. Zhonghua Jie He He Hu Xi Za Zhi. 2014;37(1):30-35.

37. Song C, Luo B, Gong L. Resveratrol reduces the apoptosis induced by cigarette smoke extract by upregulating MFN2. PLoS One. 2017; 12(4): 0175009.

38. Xuan LL, Shi J, Yao CS, et al. Vam3, a resveratrol dimer, inhibits cigarette smoke-induced cell apoptosis in lungs by improving mitochondrial function. Acta Pharmacol Sinica. 2014;35(6):779-791.

39. Chen $\mathrm{C}$, Jiang $\mathrm{X}, \mathrm{Hu} \mathrm{Y}$, Zhang Z. The protective role of resveratrol in the sodium arsenite-induced oxidative damage via modulation of intracellular GSH homeostasis. Biol Trace Element Res. 2013;155(1): 119-131.

40. Sha H, Ma Q, Jha RK, Wang Z. Resveratrol ameliorates lung injury via inhibition of apoptosis in rats with severe acute pancreatitis. Exp Lung Res. 2009;35(4):344-358.

41. Xia L, Ding F, Zhu JH, Fu GS. Resveratrol attenuates apoptosis of pulmonary microvascular endothelial cells induced by high shear stress and proinflammatory factors. Human Cell. 2011;24(3):127-133.

42. Sarma JV, Ward PA. Oxidants and redox signaling in acute lung injury. Compr Physiol. 2011;1(3):1365-1381.

43. Ozdemir OM, Gozkeser E, Bir F, Yenisey C. The effects of resveratrol on hyperoxia-induced lung injury in neonatal rats. Pediatr Neonatol. 2014;55(5):352-357.

44. Liu H, Ren J, Chen H, et al. Resveratrol protects against cigarette smokeinduced oxidative damage and pulmonary inflammation. $J$ Biochem Mol Toxicol. 2014;28(10):465-471.

45. Cerqueira AM, Khaper N, Lees SJ, Ulanova M. The antioxidant resveratrol down-regulates inflammation in an in-vitro model of Pseudomonas aeruginosa infection of lung epithelial cells. Canadian J Physiol Pharmacol. 2013;91(3):248-255. 
46. Zahlten J, Kim YJ, Doehn JM, et al. Streptococcus pneumoniae-induced oxidative stress in lung epithelial cells depends on pneumococcal autolysis and is reversible by resveratrol. J Infect Dis. 2015;211(11): $1822-1830$.

47. Zhang HX, Duan GL, Wang CN, Zhang YQ, Zhu XY, Liu YJ. Protective effect of resveratrol against endotoxemia-induced lung injury involves the reduction of oxidative/nitrative stress. Pulm Pharmacol Ther. 2014;27(2):150-155.

48. Zhang YQ, Liu YJ, Mao YF, Dong WW, Zhu XY, Jiang L. Resveratrol ameliorates lipopolysaccharide-induced epithelial mesenchymal transition and pulmonary fibrosis through suppression of oxidative stress and transforming growth factor-beta1 signaling. Clin Nutr. 2015; 34(4):752-760.

49. Ogawa EN, Ishizaka A, Tasaka S, et al. Contribution of high-mobility group box-1 to the development of ventilator-induced lung injury. Am J Respir Crit Care Med. 2006;174(4):400-407.

50. Dong WW, Liu YJ, Lv Z, et al. Lung endothelial barrier protection by resveratrol involves inhibition of HMGB1 release and HMGB1-induced mitochondrial oxidative damage via an Nrf2-dependent mechanism. Free Radical Biol Med. 2015;88(Pt B):404-416

51. Trotta V, Lee WH, Loo CY, Young PM, Traini D, Scalia S. Co-spray dried resveratrol and budesonide inhalation formulation for reducing inflammation and oxidative stress in rat alveolar macrophages. Eur $J$ Pharmaceut Sci. 2016;86:20-28

52. Chen C, Jiang X, Lai Y, Liu Y, Zhang Z. Resveratrol protects against arsenic trioxide-induced oxidative damage through maintenance of glutathione homeostasis and inhibition of apoptotic progression. Environ Mol Mutagen. 2015;56(3):333-346.

53. Lee YJ, Kim MJ, Yoon YS, Choi YH, Kim HS, Kang JL. Simvastatin treatment boosts benefits of apoptotic cell infusion in murine lung fibrosis. Cell Death Dis. 2017;8(6):e2860.

54. Du B, Shim JS. targeting epithelial-mesenchymal transition (EMT) to overcome drug resistance in cancer. Molecules. 2016;21(7):965.

55. Kage H, Borok Z. EMT and interstitial lung disease: a mysterious relationship. Curr Opin Pulm Med. 2012;18(5):517-523.

56. Abidi A, Robbe A, Kourda N, Ben Khamsa S, Legrand A. Nigella sativa, a traditional Tunisian herbal medicine, attenuates bleomycin-induced pulmonary fibrosis in a rat model. Biomed Pharmacother. 2017;90: 626-637.

57. Mouratis MA, Aidinis V. Modeling pulmonary fibrosis with bleomycin. Curr Opin Pulm Med. 2011;17(5):355-361.

58. Natalin HM, Garcia AF, Ramalho LN, Restini CB. Resveratrol improves vasoprotective effects of captopril on aortic remodeling and fibrosis triggered by renovascular hypertension. Cardiovasc Pathol. 2016;25(2) $116-119$.

59. He T, Xiong J, Nie L, et al. Resveratrol inhibits renal interstitial fibrosis in diabetic nephropathy by regulating AMPK/NOX4/ROS pathway. J Mol Med. 2016;94(12):1359-1371.

60. Rong L, Wu J, Wang W, Zhao RP, Xu XW, Hu D. Sirt 1 activator attenuates the bleomycin-induced lung fibrosis in mice via inhibiting epithelial-to-mesenchymal transition (EMT). Eur Rev Med Pharmacol Sci. 2016;20(10):2144-2150.

61. Impellizzeri D, Talero E, Siracusa R, et al. Protective effect of polyphenols in an inflammatory process associated with experimental pulmonary fibrosis in mice. Br J Nutr. 2015;114(6):853-865.

62. Akgedik R, Akgedik S, Karamanli H, et al. Effect of resveratrol on treatment of bleomycin-induced pulmonary fibrosis in rats. Inflammation. 2012;35(5):1732-1741.

63. Zeng Z, Cheng S, Chen H, et al. Activation and overexpression of Sirt1 attenuates lung fibrosis via P300. Biochem Biophys Res Commun. 2017;486(4):1021-1026

64. He X, Wang L, Szklarz G, Bi Y, Ma Q. Resveratrol inhibits paraquatinduced oxidative stress and fibrogenic response by activating the nuclear factor erythroid 2-related factor 2 pathway. J Pharmacol Exp Ther. 2012;342(1):81-90.

65. Chen D, Gao W, Wang S, Ni B, Gao Y. Critical effects of epigenetic regulation in pulmonary arterial hypertension. Cell Mol Life Sci. Epub 2017 Jun 1.
66. Boucherat O, Vitry G, Trinh I, Paulin R, Provencher S, Bonnet S. The cancer theory of pulmonary arterial hypertension. Pulm Circ. 2017; $7(2): 285-299$.

67. Nogueira-Ferreira R, Vitorino R, Ferreira R, Henriques-Coelho T. Exploring the monocrotaline animal model for the study of pulmonary arterial hypertension: a network approach. Pulm Pharmacol Ther. 2015;35:8-16

68. Paffett ML, Lucas SN, Campen MJ. Resveratrol reverses monocrotaline-induced pulmonary vascular and cardiac dysfunction: a potential role for atrogin-1 in smooth muscle. Vasc Pharmacol. 2012;56(1-2): 64-73.

69. Zhou S, Li MT, Jia YY, et al. Regulation of cell cycle regulators by SIRT1 contributes to resveratrol-mediated prevention of pulmonary arterial hypertension. BioMed Res Int. 2015;2015:762349.

70. Wilson DN, Schacht SE, Al-Nakkash L, Babu JR, Broderick TL. Resveratrol prevents pulmonary trunk remodeling but not right ventricular hypertrophy in monocrotaline-induced pulmonary hypertension. Pathophysiology. 2016;23(4):243-250.

71. Yang DL, Zhang HG, Xu YL, et al. Resveratrol inhibits right ventricular hypertrophy induced by monocrotaline in rats. Clin Exp Pharmacol Physiol. 2010;37(2):150-155.

72. Chun C, Yang W, Xueding C, et al. Resveratrol downregulates acute pulmonary thromboembolism-induced pulmonary artery hypertension via p38 mitogen-activated protein kinase and monocyte chemoattractant protein-1 signaling in rats. Life Sci. 2012;90(19-20):721-727.

73. Luo Y, Zhang B, Dong HY, et al. Prevention of hypoxic pulmonary hypertension by hypoxia-inducible expression of p27 in pulmonary artery smooth muscle cells. Gene Ther. 2014;21(8):751-758.

74. Xu D, Li Y, Zhang B, et al. Resveratrol alleviate hypoxic pulmonary hypertension via anti-inflammation and anti-oxidant pathways in rats. Int J Med Sci. 2016;13(12):942-954.

75. Chen B, Xue J, Meng X, Slutzky JL, Calvert AE, Chicoine LG. Resveratrol prevents hypoxia-induced arginase II expression and proliferation of human pulmonary artery smooth muscle cells via Aktdependent signaling. Am J Physiol Lung Cell Mol Physiol. 2014;307(4): L317-L325.

76. Yu L, Tu Y, Jia X, et al. Resveratrol protects against pulmonary arterial hypertension in rats via activation of silent information regulator 1. Cell Physiol Biochem. 2017;42(1):55-67.

77. Liu B, Luo XJ, Yang ZB, et al. Inhibition of NOX/VPO1 pathway and inflammatory reaction by trimethoxystilbene in prevention of cardiovascular remodeling in hypoxia-induced pulmonary hypertensive rats. $J$ Cardiovasc Pharmacol. 2014;63(6):567-576.

78. Gao G, Wang X, Qin X, et al. Effects of trimethoxystilbene on proliferation and apoptosis of pulmonary artery smooth muscle cells. Cell Biochem Biophys. 2012;64(2):101-106.

79. Ko JC, Syu JJ, Chen JC, et al. Resveratrol enhances etoposide-induced cytotoxicity through down-regulating ERK1/2 and AKT-mediated $\mathrm{X}$-ray repair cross-complement group 1 (XRCC1) protein expression in human non-small-cell lung cancer cells. Basic Clin Pharmacol Toxicol. 2015;117(6):383-391.

80. Luo H, Yang A, Schulte BA, Wargovich MJ, Wang GY. Resveratrol induces premature senescence in lung cancer cells via ROS-mediated DNA damage. PLoS One. 2013;8(3):e60065.

81. Lucas IK, Kolodziej H. Trans-resveratrol induces apoptosis through ROS-triggered mitochondria-dependent pathways in A549 human lung adenocarcinoma epithelial cells. Planta Med. 2015;81(12-13): 1038-1044.

82. Shi Q, Geldenhuys W, Sutariya V, Bishayee A, Patel I, Bhatia D. CArG-driven GADD45alpha activated by resveratrol inhibits lung cancer cells. Genes Cancer. 2015;6(5-6):220-230.

83. Sun L, Chen B, Jiang R, Li J, Wang B. Resveratrol inhibits lung cancer growth by suppressing M2-like polarization of tumor associated macrophages. Cell Immunol. 2017;311:86-93.

84. Kimura Y, Sumiyoshi M. Resveratrol prevents tumor growth and metastasis by inhibiting lymphangiogenesis and M2 macrophage activation and differentiation in tumor-associated macrophages. Nutr Cancer. 2016;68(4):667-678. 
85. Wang X, Wang D, Zhao Y. Effect and mechanism of resveratrol on the apoptosis of lung adenocarcinoma cell line A549. Cell Biochem Biophys. 2015;73(2):527-531.

86. Yin HT, Tian QZ, Guan L, Zhou Y, Huang XE, Zhang H. In vitro and in vivo evaluation of the antitumor efficiency of resveratrol against lung cancer. Asian Pac J Cancer Prev. 2013;14(3):1703-1706.

87. Li X, Wang D, Zhao QC, Shi T, Chen J. Resveratrol inhibited non-small cell lung cancer through inhibiting STAT-3 signaling. Am J Med Sci. 2016;352(5):524-530.

88. Li W, Ma X, Li N, et al. Resveratrol inhibits Hexokinases II mediated glycolysis in non-small cell lung cancer via targeting Akt signaling pathway. Exp Cell Res. 2016;349(2):320-327.

89. Zhang L, Dai F, Sheng PL, Chen ZQ, Xu QP, Guo YQ. Resveratrol analogue 3,4,4'-trihydroxy-trans-stilbene induces apoptosis and autophagy in human non-small-cell lung cancer cells in vitro. Acta Pharmacol Sinica. 2015;36(10):1256-1265.

90. Bai T, Dong DS, Pei L. Synergistic antitumor activity of resveratrol and miR-200c in human lung cancer. Oncol Rep. 2014;31:2293-2297.

91. Yang Q, Xu E, Dai J, et al. A novel long noncoding RNA AK001796 acts as an oncogene and is involved in cell growth inhibition by resveratrol in lung cancer. Toxicol Appl Pharmacol. 2015;285(2):79-88.

92. Bae S, Lee EM, Cha HJ, et al. Resveratrol alters microRNA expression profiles in A549 human non-small cell lung cancer cells. Mol Cells. 2011;32(3):243-249.

93. Zhu Y, He W, Gao X, et al. Resveratrol overcomes gefitinib resistance by increasing the intracellular gefitinib concentration and triggering apoptosis, autophagy and senescence in PC9/G NSCLC cells. Sci Rep. 2015;5(1):17730.

94. Hu S, Li X, Xu R, et al. The synergistic effect of resveratrol in combination with cisplatin on apoptosis via modulating autophagy in A549 cells. Acta Biochim Biophys Sinica. 2016;48(6):528-535.

95. Gu S, Chen C, Jiang X, Zhang Z. ROS-mediated endoplasmic reticulum stress and mitochondrial dysfunction underlie apoptosis induced by resveratrol and arsenic trioxide in A549 cells. Chem Biol Interact. 2016; 245:100-109.

96. Gu S, Chen C, Jiang X, Zhang Z. Resveratrol synergistically triggers apoptotic cell death with arsenic trioxide via oxidative stress in human lung adenocarcinoma A549 cells. Biol Trace Elem Res. 2014; 163(1-2):112-123.

97. Nie P, Hu W, Zhang T, Yang Y, Hou B, Zou Z. Synergistic induction of erlotinib-mediated apoptosis by resveratrol in human non-small-cell lung cancer cells by down-regulating survivin and up-regulating PUMA. Cell Physiol Biochem. 2015;35(6):2255-2271.
98. Lachenmeier DW, Godelmann R, Witt B, Riedel K, Rehm J. Can resveratrol in wine protect against the carcinogenicity of ethanol? A probabilistic dose-response assessment. Int J Cancer. 2014;134(1): 144-153.

99. Wan ZL, Wang JM, Wang LY, Yuan Y, Yang XQ. Complexation of resveratrol with soy protein and its improvement on oxidative stability of corn oil/water emulsions. Food Chem. 2014;161:324-331.

100. Walle T. High absorption but very low bioavailability of oral resveratrol in humans. Drug Metab Dispos. 2004;32(12):1377-1382.

101. Delmas D, Aires V, Limagne E, et al. Transport, stability, and biological activity of resveratrol. Ann NY Acad Sci. 2011;1215(1):48-59.

102. Vian MA, Tomao V, Gallet S, Coulomb PO, Lacombe JM. Simple and rapid method for cis- and trans-resveratrol and piceid isomers determination in wine by high-performance liquid chromatography using Chromolith columns. J Chromatogr A. 2005;1085(2): 224-229.

103. Zupančič Š, Lavrič Z, Kristl J. Stability and solubility of transresveratrol are strongly influenced by $\mathrm{pH}$ and temperature. Eur J Pharm Biopharm. 2015;93:196-204.

104. Goldberg DM, Yan J, Soleas GJ. Absorption of three wine-related polyphenols in three different matrices by healthy subjects. Clin Biochem. 2003;36(1):79-87.

105. Francioso A, Mastromarino P, Masci A, d'Erme M, Mosca L. Chemistry, stability and bioavailability of resveratrol. Med Chem. 2014; 10(3):237-245.

106. Reis S, Neves AR, Lúcio M, Martins S, Lima JLC. Novel resveratrol nanodelivery systems based on lipid nanoparticles to enhance its oral bioavailability. Int J Nanomedicine. 2013;8:177-187.

107. Lucas-Abellan C, Mercader-Ros MT, Zafrilla MP, Fortea MI, Gabaldon JA, Nunez-Delicado E. ORAC-fluorescein assay to determine the oxygen radical absorbance capacity of resveratrol complexed in cyclodextrins. J Agric Food Chem. 2008;56(6): 2254-2259.

108. Wegiel LA, Mauer LJ, Edgar KJ, Taylor LS. Crystallization of amorphous solid dispersions of resveratrol during preparation and storage-impact of different polymers. J Pharm Sci. 2013;102(1): $171-184$

109. Hung CF, Chen JK, Liao MH, Lo HM, Fang JY. Development and evaluation of emulsion-liposome blends for resveratrol delivery. J Nanosci Nanotech. 2006;6(9):2950-2958.

110. Bu L, Gan LC, Guo XQ, et al. Trans-resveratrol loaded chitosan nanoparticles modified with biotin and avidin to target hepatic carcinoma. Int J Pharm. 2013;452(1-2):355-362.
Drug Design, Development and Therapy

\section{Publish your work in this journal}

Drug Design, Development and Therapy is an international, peerreviewed open-access journal that spans the spectrum of drug design and development through to clinical applications. Clinical outcomes, patient safety, and programs for the development and effective, safe, and sustained use of medicines are the features of the journal, which

\section{Dovepress}

has also been accepted for indexing on PubMed Central. The manuscript management system is completely online and includes a very quick and fair peer-review system, which is all easy to use. Visit http://www.dovepress.com/testimonials.php to read real quotes from published authors. 